\title{
Management of some extra-peritoneal rectal injuries without fecal diversion may be feasible, but high-quality evidence is still needed
}

\author{
R. W. Schroll ${ }^{1}$
}

Received: 29 November 2018 / Accepted: 30 November 2018 / Published online: 6 December 2018

(c) Springer Nature Switzerland AG 2018

Although relatively rare, traumatic rectal injuries have long been known to have high morbidity and mortality rates, with infectious complications reported up to $19 \%$ and mortality rates of up to $11 \%[1,2]$. Because of the serious nature of these injuries, it is important to attempt to understand and define optimal management strategies. Evidence-based consensus has been difficult to achieve, partially due to the complicated nature of injury location that may affect diagnosis, management, and outcomes-intra-peritoneal vs extraperitoneal vs combined-and the complex options that have been advocated as management strategies over the years, including resection vs primary repair, plus/minus diverting stoma formation, plus/minus distal rectal irrigation, and for extra-peritoneal injuries, plus/minus pre-sacral drainage. In addition, due to the rarity of their occurrence, most previous studies have been limited to small, single-center, retrospective reviews.

In the study published in this issue of Techniques in Coloproctology, Gash and colleagues have published a noteworthy achievement-using 2 years of National Trauma Data Bank (NTDB) data, they have published a very large study of rectal trauma management and outcomes. Of 1.7 million patients, they found a $0.1 \%$ rectal injury rate, resulting in an analysis which includes 1472 adult patients with rectal injury [3]. Perhaps unsurprisingly, the majority of patients were young, male, and had a penetrating injury mechanism. Patients with penetrating mechanism were younger, more likely to be male, and had a lower mean injury severity score than those with blunt mechanism. Understandably, they were also more likely to have an intra-peritoneal location of their rectal injuries and to be managed operatively compared to patients with blunt mechanism.

\section{R. W. Schroll}

rschroll@tulane.edu

1 Department of Surgery, Tulane University School of Medicine, 1430 Tulane Ave, \#8622, New Orleans, LA 70112, USA
A little over half of the study patients (53\%) has isolated extra-peritoneal injuries, and although fecal diversion has long been considered standard practice in management of rectal injuries [2], in this study, $63 \%$ of patients with extraperitoneal injury were managed without diverting ostomy (though the authors' abstract says $63 \%$ of patients were managed by resection/repair without stoma, upon close reading of the study is seems that this includes all patients managed without diverting stoma, including those who were managed "non-operatively" without primary resection or repair). Despite having a similar injury severity score, the non-diverted patients had a lower morbidity rate and shorter hospital LOS than the 37\% who were diverted. This finding suggests that selected isolated extra-peritoneal rectal injuries may be successfully managed without requiring diversion; a practice that is supported by some smaller single-center studies [4-6], but which has not been reproduced by a larger scale study until now. It may be that conducting fecal diversion for patients with selected extra-peritoneal injuries might actually increase morbidity due to the addition of an abdominal operation; however, we must be careful not to draw conclusions with incomplete data or assume that correlation is the same as causation. As the authors point out, the decision to create a diverting ostomy is a complex one influenced by many factors that might cause poor healing and leakage of the repair/anastomosis, such as the grade of the injury, delayed diagnosis, incidence of hemorrhagic shock or septic shock, or other factors that may compromise intestinal perfusion. These factors are not well accounted for in the NTDB data set, so it may be that this group is suffering from some selection bias; in patients with extra-peritoneal injury, the same risk factors that led surgeons to decide to create a diverting ostomy may have also contributed to poorer outcomes. Patients with intra-peritoneal injuries were more likely to be managed with diverting ostomy than not $(64 \%$ vs $36 \%$ ) and the study authors found no significant differences in morbidity or mortality between these two groups on crude analysis. On multi-variate analysis, however, stoma 
formation overall was associated with significantly lower mortality than management without fecal diversion. The authors conclude that surgeons should have a low threshold for consideration of an ostomy for significantly destructive or intra-peritoneal rectal injuries; indeed, this is a conclusion that has been advocated previously based on small studies and is re-demonstrated here with a much larger data set.

Interestingly, in this study, $46 \%$ of patients with extraperitoneal injury and $27 \%$ of patients with intra-peritoneal injury were managed without ANY surgical interventionneither resection, nor repair, nor ostomy creation-including $31 \%$ of patients with a penetrating injury mechanism. This is a higher incidence of non-surgical management of rectal injury than has been previously reported [1], particularly in the intra-peritoneal injury group, which begs the question-how exactly were these rectal injuries diagnoses and managed? Several possibilities come to mind that might explain these findings. Were these injuries so minor (AAST grade 1 -hematoma only) that they were felt by the treating physician not to require resection or repair? It certainly seems likely that if these injuries were truly successfully managed non-operatively, they must have been low grade, particularly the intra-peritoneal group. It is also possible that these patients were indeed managed surgically, but were not included in the operative management group due to limitations of the study design and the data available in the NTDB database. For instance, high intra-peritoneal rectal injuries managed by recto-sigmoid resection might have been miscoded as colon resection rather than rectal resection and, therefore, not included in the operative management group. As the authors point out, rectal injuries can be difficult to diagnose and are often missed at initial presentation; were some injuries identified in a delayed manner and managed by percutaneous drainage procedures only but then included in the non-surgical management group? Could outcomes have been affected by the incidence of pre-sacral drainage and/ or distal rectal washout, both of which are procedures that have comprised standard management for rectal injury by many surgeons, and may have been conducted on a number of patients in this study including those in the non-operative group, but are not captured by the NTDB? Previous studies that have reported high rates of non-operative management in extra-peritoneal rectal injuries have included patients who have undergone both pre-sacral drainage and distal rectal washout in the non-operative category, making the term "non-operative" somewhat of a misnomer [1]. Unfortunately, the limitations imposed by retrospective analysis of large databanks such as the NTDB mean that we do not have answers to these questions, since many of these variables simply cannot be accounted for.

In the end, as with any large database study, this paper is quite interesting in that it raises many possibilities for future study directions. Gash and colleagues have put together the largest study yet of patients with traumatic rectal injuries, which is quite important when studying such an uncommon condition. They demonstrated the rarity of rectal trauma and the wide variability of presentation and management that occurs in trauma centers across the United States. Their study suggests that it may be feasible to manage select isolated extra-peritoneal rectal injuries without fecal diversion, but lacks specific details necessary to definitively provide evidence for this change in practice. Hopefully, further studies, such as an ongoing multi-center prospective trial by the AAST, will further elucidate the answer to this question.

\section{Compliance with ethical standards}

Conflict of interest The author declares she had no conflicts of interest.

Ethical approval This article does not contain any studies with human participants by the author.

\section{References}

1. Ahern DP, Kelly ME, Courtney D, Rausa E, Winter DC (2017) The management of penetrating rectal and anal trauma: a systematic review. Injury 48(6):1133-1138

2. Borsarge PL, Como JJ, Fox N, Falck-Ytter Y, Haut ER, Dorion HA, Patel NJ, Rushing A, Raff LA, McDonald AA, Robinson BR, McGwin G Jr, Gonzalez RP (2016) Management of penetrating extraperitoneal rectal injuries: an Eastern Association for the Surgery of Trauma practice management guideline. J Trauma Acute Care Surg 80(3):546-551

3. Gash KJ, Suradkar K, Kiran R (2018) Rectal trauma injuries: outcomes from the US National Trauma Data Bank. Tech Coloproctol. https://doi.org/10.1007/s10151-018-1856-4

4. Gonzalez RP, Phelan H, Hassan M, Ellis CN, Rodning CB (2006) Is fecal diversion necessary for nondestructive penetrating extraperitoneal rectal injuries? J Trauma 61(4):815-819

5. Levine JH, Longo WE, Pruitt C, Mazuski JE, Shapiro MJ, Durham RM (1996) Management of selected rectal injuries by primary repair. Am J Surg 172(5):575-578

6. Weinberg JA, Fabian TC, Magnotti LJ, Minard G, Bee TK, Edwards N, Claridge JA, Croce MA (2006) Penetrating rectal trauma: management by anatomic distinction improves outcome. J Trauma 60(3):508-513 